\title{
TTR
}

Traduction, terminologie, rédaction

\section{Boucler la boucle : réflexions sur un trajet traductologique en spirale}

\section{Judith Woodsworth}

Volume 30, numéro 1-2, 1er semestre-2e semestre 2017

La traductologie en mouvement

Translations Studies: A Forward-Moving Discipline

URI : https://id.erudit.org/iderudit/1060017ar

DOI : https://doi.org/10.7202/1060017ar

Aller au sommaire du numéro

Éditeur(s)

Association canadienne de traductologie

ISSN

0835-8443 (imprimé)

1708-2188 (numérique)

Découvrir la revue

Citer cet article

Woodsworth, J. (2017). Boucler la boucle : réflexions sur un trajet

traductologique en spirale. TTR, 30(1-2), 39-59.

https://doi.org/10.7202/1060017ar
Résumé de l'article

Cet article retrace le cheminement intellectuel de l'auteure, depuis la fondation de l'Association canadienne de traductologie jusqu'au présent, évolution qui reflète, en quelque sorte, celle de la discipline émergente de la traductologie en général. Depuis le premier article qu'elle publie dans TTR, portant sur les traducteurs et écrivains, elle ne cesse de s'intéresser au sujet traduisant, ce qui débouche sur une histoire des traducteurs, suivie de différentes études d'éminents écrivains-traducteurs. L'article propose quelques mini-portraits de ces écrivains - Paul Valéry, Bernard Shaw, Gertrude Stein, Paul Auster, notamment - afin de faire ressortir leur motivation à traduire et les différentes façons dont ils conçoivent et manipulent la traduction. 


\title{
Boucler la boucle : réflexions sur un trajet traductologique en spirale
}

\author{
Judith Woodsworth
}

Université Concordia

\section{Résumé}

Cet article retrace le cheminement intellectuel de l'auteure, depuis la fondation de l'Association canadienne de traductologie jusqu'au présent, évolution qui reflète, en quelque sorte, celle de la discipline émergente de la traductologie en général. Depuis le premier article qu'elle publie dans TTR, portant sur les traducteurs et écrivains, elle ne cesse de s'intéresser au sujet traduisant, ce qui débouche sur une histoire des traducteurs, suivie de différentes études d'éminents écrivains-traducteurs. L'article propose quelques mini-portraits de ces écrivains - Paul Valéry, Bernard Shaw, Gertrude Stein, Paul Auster, notamment - afin de faire ressortir leur motivation à traduire et les différentes façons dont ils conçoivent et manipulent la traduction.

Mots-clés : traducteurs et traductrices, écrivains-traducteurs, Valéry, Shaw, Stein, Auster

\section{Abstract}

This article seeks to outline my intellectual path from the launch of the Canadian Association for Translation Studies to the present, a development that in many ways reflects the evolution of the emerging discipline itself. Since my first article in TTR on translators and writers, I have always maintained an interest in the translator. This resulted in a history of translators, followed by studies on eminent writer-translators. Included in this articles are miniportraits of some of the authors I have examined-Paul Valéry, Bernard Shaw, Gertrude Stein, and Paul Auster principally-illustrating their motivation to translate and the various ways in which they conceptualize translation and manipulate the translation process.

Keywords: the translator, writer-translators, Valéry, Shaw, Stein, Auster

Le présent article se veut une sorte d'autobiographie intellectuelle, réponse à l'invitation de participer à ce numéro marquant le $30^{\mathrm{e}}$ anniversaire de TTR. En sollicitant ma contribution, on m'a demandé, en premier lieu, de me pencher sur l'article que j’avais signé 
pour le premier numéro de la revue et de tracer ensuite l'évolution de mon champ d'expertise et de mon propre trajet théorique depuis ce temps. En feuilletant le texte «Traducteurs et écrivains : vers une redéfinition de la traduction littéraire» que j'avais rédigé en 1988 et en jetant un coup d'œil sur mes travaux récents, par exemple, mon ouvrage Telling the Story of Translation: Writers Who Translate (2017), j'ai l'impression, au premier abord, de ne pas avoir fait beaucoup de chemin en trente ans. Tout bien réfléchi cependant, je pourrais faire le constat inverse : je suis arrivée à l'état actuel de mes recherches après avoir suivi une longue route, quelque peu sinueuse, en empruntant parfois des voies pas encore bien déblayées, mais non moins passionnantes, de l'histoire de la traduction. Voici donc l'histoire d'une historienne de la traduction, ponctuée de mini-portraits d'écrivainstraducteurs que j'ai étudiés. Le regard en arrière que je jette sur ma pensée et sur mes travaux révélera un cheminement qui reflète, à bien des égards, celui de la discipline émergente de la traductologie ${ }^{1}$.

\section{D’une activité « souterraine " à une discipline sui generis}

On est au milieu des années 1980. Au Canada, le domaine de la traduction est en plein essor : après l'adoption de lois et politiques linguistiques tant au niveau fédéral qu'au niveau provincial, l'activité de traduction s'intensifie. Exercée par des milliers de professionnels, elle est enseignée dans un nombre croissant d'universités, et même de collèges, du pays. Dans les organismes professionnels et les associations universitaires comme l'Association canadienne des écoles de traduction, on s'interroge sur les différents aspects de la traduction, y compris sa pédagogie et les moyens de renforcer les recherches en traduction. La traduction est examinée par des spécialistes sous divers points de vue, de sorte qu'un nouveau champ de connaissance se constitue peu à peu. James Holmes avait déjà fait la cartographie d'une discipline qu'il baptisait «translation studies» (2004 [1972]). Des théoriciens de la traduction aux Pays-Bas, en Belgique aussi bien qu'à Tel-Aviv et en Grande-Bretagne se prononcent sur ce qui deviendra la «manipulation school», la théorie des polysystèmes, entre autres. Un centre de recherche sur les traductions littéraires s'établit à Göttingen en Allemagne. Bref, diverses approches se manifestent, qui dépassent les considérations purement linguistiques et qui se transformeront en «tournant culturel» (Bassnett et Lefevere, 1990).

1. Je tiens à remercier Marthe Catry-Verron pour sa relecture attentive de cet article, ainsi que pour ses encouragements de longue date. 
En France, Antoine Berman publie L'Épreuve de l'étranger (1984), ouvrage pionnier qui trace l'histoire de la traduction dans l'Allemagne romantique et qui, en outre, s'annonce dans l'introduction comme un «manifeste» de la traduction. C'est dans ce texte liminaire que Berman signale le statut secondaire de la traduction et lance un appel à l'action :

D’une part, la traduction est demeurée une activité souterraine, cachée, parce quelle ne s'énonçait pas elle-même. D'autre part, elle est restée largement «impensée» comme telle, parce que ceux qui en traitaient avaient tendance à l'assimiler à autre chose : à de la (sous-) littérature, à de la (sous-) critique, à de la linguistique appliquée. (1984, p. 11)

Au Canada, on suit de près ces tendances, on y contribue, et on constate qu'il reste beaucoup à faire. Un pas important est franchi en 1987, lorsqu'on crée une "société savante», association distincte vouée à la recherche en traduction ${ }^{2}$. À la réunion de fondation, tout en énonçant de grands principes et de grandes lignes, on débat aussi un problème de traduction, ou plutôt de terminologie, lorsqu'on choisit comme désignation de l'association naissante le néologisme français «traductologie», dont l'équivalent anglais «translation studies» avait été mis en circulation par Holmes, notamment. L'Association canadienne de traductologie/Canadian Association for Translation Studies (ACT/CATS) a fait ses preuves; âgée maintenant de plus de trente ans, elle continue à rassembler ceux et celles qui s'intéressent aux dimensions théoriques de la traduction. C'est un regroupement vital de jeunes chercheurs et de chercheurs chevronnés, en provenance des quatre coins du Canada et de plusieurs autres régions du monde.

\section{Une société savante mérite sa revue savante}

Jean-Marc Gouanvic, alors professeur à l'Université du Québec à Trois-Rivières, a la brillante idée de lancer une revue, TTR. Peu de temps après, l'ACT vote l'adoption de la nouvelle née comme revue officielle de l'association. Lorsque Jean-Marc Gouanvic est embauché comme professeur de traduction à l'Université Concordia, la revue déménage avec lui, et le directeur fondateur et la présidente fondatrice se retrouvent dans le même département : l'association est partie comme sur des roulettes.

2. Jean Delisle (s.d.) trace l'historique de l'Association canadienne de traductologie en reprenant le rapport sur sa genèse et sa fondation que j'ai fait paraître, en tant que présidente fondatrice, dans le premier numéro du Bulletin de l'ACT paru à l'automne de 1987. 
Pour le premier numéro de $T T R$, auquel on a donné le beau titre «Traduction et culture(s)» (Gouanvic, 1988), le directeur avait audacieusement sollicité des textes de grands chercheurs - Antoine Berman, par exemple, qui venait d'offrir au public son important ouvrage L'Épreuve de l'etranger, et Sherry Simon, déjà en voie de devenir l'une des sommités du monde de la traductologie. J'étais parmi les chercheurs de moindre stature à qui il avait également demandé une contribution. J'enseignais depuis à peine une demi-décennie, ayant occupé auparavant un poste de traductrice au Bureau de la traduction à Ottawa. Affectée au ministère de la Défense nationale, j'avais été confrontée à des textes aussi techniques qu'arides portant sur les véhicules blindés et les armes de combat, textes qui n'avaient rien à voir, à vrai dire, avec ma formation littéraire antérieure. En effet, je venais tout juste de terminer mon doctorat en littérature française. Dans ma thèse (Woodsworth, 1977), je m'étais interrogée sur les liens entre une lignée de poètes français importants - Baudelaire, Mallarmé, Valéry - et Poe, poète «maudit» américain. Avant même de me consacrer à la tâche de traductrice, donc, la traduction et les échanges littéraires interculturels figuraient au cœur de ma réflexion.

Rendue à l'université, je me suis vu attribuer des cours pratiques, dont la traduction littéraire, mais aussi toute une gamme de cours pragmatiques - de la traduction scientifique et technique à l'adaptation publicitaire. On m'avait confié également un cours intitulé «Théories de la traduction $»^{3}$, que j'alimentais en parcourant les rayons plutôt mal garnis de la bibliothèque. Ce que je remarquais, à l'époque, c'était qu'il manquait une vue d'ensemble de la traduction littéraire. Les traducteurs littéraires eux-mêmes réfléchissaient depuis toujours sur leur art, mais ils le faisaient de manière anecdotique et, surtout, normative ou prescriptive, le pendule oscillant entre une approche fidèle et une approche libre. Les théories linguistiques apportaient une dimension de plus en plus scientifique, mais l'accent était mis sur le texte et les obstacles à la traduction découlant des différences entre les langues. D'autres théoriciens, plus axés sur la littérature, avaient préconisé un examen de la traduction comme produit culturel et étudiaient son insertion dans le système littéraire de la culture

3. Il est à noter qu'on avait retenu le terme théories au pluriel, trouvaille heureuse de la part de mes collègues du Département d'études françaises, qui avaient conçu le programme de traduction avant que j'arrive, sans trop savoir, par contre, en quoi un tel cours consisterait. 
cible. Une lacune, me semble-t-il, restait à combler : celle du sujet traduisant, qui serait justement la visée de mon article pour le premier numéro de $T T R$, et qui resterait mon sujet de prédilection tout au long de ma carrière.

Deux choses ont attiré mon attention : en premier lieu, le discours des traducteurs sur la traduction qui, selon mes observations, s'avérait particulièrement métaphorique et, en deuxième lieu, le comportement des traducteurs qui étaient aussi écrivains. Dans ce premier article pour TTR, donc, j'amorce un début de «florilège » de métaphores de la traduction ${ }^{4}$. Je cite un certain nombre d'exemples dans lesquels la traduction est représentée de façon négative aussi bien que positive. Voici, parmi les citations que je préfère, le beau mot de Madame de Staël : «Une musique composée pour un instrument n'est point exécutée avec succès sur un instrument d'un autre genre» (cité par Berman, 1985, p. 62; repris par Woodsworth, 1988, p. 117). Ce motif musical, comme celui de la peinture, revient souvent pour figurer l'idée de perte en traduction. À ce genre de représentation, on peut ajouter les propos qui soulignent la nature déloyale du traducteur (reliée au célèbre adage traduttore, traditore), mais qui insiste toutefois sur l'apport de cette espèce de «double agent». Comme l'éminent traducteur canadien Philip Stratford l'a fait remarquer :

As literary contrabandier the translator [...] is by necessity a man of divided allegiances, neither flesh nor fowl, a lonely, shadowy character, mistrusted by everyone. And probably envied a little in a covert way, too, for, more positively, he stands for freedom, risk, excitement and adventure. (cité par Woodsworth, 1988, p. 118)

Finalement, l'heureuse formule de Jackson Mathews, traducteur de Paul Valéry, qui conçoit le traducteur comme un amant, thème auquel je reviendrai plus loin à propos de Paul Auster: "Being faithful without seeming to be-that is one of the "secrets", one of the special joys of this labor. A translator should make a good lover» (cité par Woodsworth, 1988, p. 119).

4. Dans Les tours de Babel, Berman déplore l'absence d'un tel florilège qui nous en apprendrait plus sur la traduction que des ouvrages spécialisés (1985, p. 63). Luce Guillerm (1980) et Theo Hermans (1985) avaient examiné le même phénomène, qui a été l'objet de maintes études depuis. J'ai moi-même étudié les métaphores de la traduction, sujet d'une communication puis d'une publication (Woodsworth, 1990). Jean Delisle, enfin, est celui qui comble le plus la lacune identifiée par Berman, dans son ouvrage La traduction en citations (2017 [2007]), dont le sous-titre «Florilège» et l'épigraphe (p.xv) sont empruntés à Berman. 
La deuxième partie de l'article porte sur la rencontre de Baudelaire et Edgar Poe, et l'influence profonde de Poe sur lui, ainsi que sur d'autres poètes du calibre de Mallarmé et de Valéry. C'est un cas extrême de traduction littéraire qui sert d'excellent point de départ à toute discussion sur la motivation de traduire. Le statut de grand écrivain que Poe a atteint, non seulement en France mais ailleurs en Europe, alors qu'il était considéré comme un écrivain relativement mineur dans son propre pays, est attribuable au travail de Baudelaire en tant que traducteur. En effet, Baudelaire proclame, tel un missionnaire, «[i]1 faut, c'est-à-dire que je désire qu'Edgar Poe qui n'est pas grand'chose en Amérique, devienne un grand homme pour la France» (1932, p. 352-353; cité par Woodsworth, 1988). Et Baudelaire a bien atteint son objectif. Voilà un bel exemple de l'acte de traduction comme hommage ou «monument $»^{5}$.

\section{Ne méprisez jamais le traducteur: construire l'histoire de la traduction}

C'est le célèbre poète Alexandre Pouchkine, reconnu comme le père de la littérature russe, traducteur d'auteurs français tels que Voltaire, et d'auteurs italiens, latins et grecs, qui nous conseille de ne pas mépriser le traducteur, "cheval de poste de la civilisation humaine» (cité par Delisle et Woodsworth, 2014, p. xx).

Le hasard a voulu que je participe aux travaux qui consisteraient à jeter une nouvelle lumière sur les traducteurs et traductrices dans l'histoire. C'est à Jean Delisle que je dois mes aventures dans l'histoire de la traduction. Le moment propice est venu en 1990 lorsque Jean Delisle se préparait à entreprendre au sein de la Fédération internationale des traducteurs (FIT) un projet ambitieux, celui de ressusciter un projet qui était presque aussi vieux que la FIT ellemême. En effet, l'idée d'une «histoire universelle de la traduction» remontait au début des années 1960, ayant été proposée à la FIT par le Hongrois György Radó. Toutefois, les années avaient passé sans qu'on soit capable de réaliser un projet d'une telle envergure. Assumant la présidence du nouveau Comité pour l'histoire de la traduction, Jean Delisle proposa au Congrès mondial de la FIT tenu à Belgrade en 1990 de diriger un projet de publication qui irait dans le

5. Rappelons que Stéphane Mallarmé, qui traduira les poèmes de Poe, place en début de son recueil de traductions l'un de ses propres poèmes, justement un poème en hommage au poète américain, «Le tombeau d'Edgar Poe». Voir aussi Galli (2012). 
sens du projet de Radó et dont le but serait de sortir les traducteurs et traductrices de l'ombre, en faisant connaître leur apport à l'évolution intellectuelle et culturelle de l'humanité. Désignée vice-présidente du Comité pour l'histoire de la traduction, je me suis lancée dans cette vaste entreprise.

S'inspirant encore une fois de Berman, qui proclame dans le même ouvrage pionnier que "la constitution d'une histoire de la traduction est la première tâche d'une théorie moderne de la traduction" (1984, p. 12), le Comité pour l'histoire de la traduction de la FIT s'était fixé comme objectif de relever ce défi sans pour autant entreprendre l'histoire encyclopédique envisagée avec tant d'optimisme par Radó. L'étude du passé de notre profession, nous semblait-il, concourrait à «légitimer la traduction en tant que discipline autonome, capable de se définir elle-même, de tenir un discours sui generis» (Delisle et Woodsworth, 2014, p. xxi). La discipline s'était déjà institutionnalisée au moyen de la création d'une association qui avait comme but de promouvoir la recherche; on lui avait donné un nom, celui de «traductologie» en français, «translation studies» en anglais. Le moment était venu de se mettre à la construction d'une histoire de la traduction.

Comme nous l'avons souligné dans l'Avant-propos à la première édition des Traducteurs dans l'histoire, nous avons conçu, à la place d'une histoire exhaustive, "un panorama sélectif et thématique des principaux rôles joués par les traducteurs au fil des siècles» (ibid., p. 2; italique dans l'original). Grâce à la collaboration d'une cinquantaine de chercheurs provenant d'une vingtaine de pays, nous avons pu peindre un tableau assez large et diversifié des contributions des traductrices et traducteurs à des domaines aussi variés que la diffusion des sciences, la création de dictionnaires et la propagation des religions. Les multiples perspectives apportées par cette équipe de chercheurs nous permettaient de dépasser un simple cadre biographique, pour tenir compte de l'identité et du contexte sociaux, culturels, religieux, politiques des traducteurs et traductrices.

Le livre a eu un certain succès; il continue à en avoir. Différentes éditions et versions se succédèrent. Publié simultanément en anglais et en français en 1995, il fut traduit en portugais au Brésil, en espagnol en Colombie, et par la suite en arabe et en roumain. En nous intéressant aux personnes qui traduisent, «aux agents plutôt qu'au produit final ou au processus », comme le précise la préface à l'édition la plus récente de l'ouvrage, nous faisions un choix qui «n'allait pas de 
soi dans une discipline qui s'était jusqu'alors concentrée sur le texte» (ibid., pp.xiii-xiv). De nos jours, cette approche est courante. Le théoricien anglo-finlandais Andrew Chesterman en vient même à proclamer l'existence de la sous-discipline «translator studies» (2009; c'est nous qui soulignons).

\section{Pourquoi traduire?}

Après mêtre adonnée pendant environ cinq ans à ce survol passionnant des vastes horizons de la traduction à travers le monde et depuis des millénaires, je suis revenue à mes intérêts plus ciblés. Je me suis remise à penser au cas de Baudelaire et de ses successeurs, cas où la "pulsion du traduire», pour encore emprunter des termes à Berman (1984, p. 21), est primordiale. La question qui se pose d'emblée est la suivante : pourquoi traduire? Qu'est-ce qui motive un écrivain à faire de la traduction, et quelle est la nature de ce type d'«affinité élective »? Comme questionnement corollaire : quels sont les rapports de l'écrivain-traducteur à l'auteur et au texte d'origine? S'agit-il de rapports différents de ceux qu'un «simple» traducteur pourrait avoir dans la même situation? ${ }^{6}$ Autrement dit, les écrivains-traducteurs se positionnent-ils différemment à l'égard de l'acte de traduire? Ces questions, qui relèvent de ce qu'on appelle de nos jours l'«agentivité» du traducteur, méritent qu'on les regarde de plus près.

Même si je n'ai pas tenu la promesse exprimée dans la conclusion de l'article de 1988, à savoir d'entreprendre une étude approfondie sur les différentes attitudes et préoccupations des traducteurs canadiens (Woodsworth, 1988, p. 125), j’ai quand même poursuivi ma réflexion sur certains grands traducteurs-écrivains, ce qui, au terme de mon long détour vers l'histoire de la traduction, a abouti à des études de cas : sur Paul Valéry (Woodsworth, 2000a), sur Bernard Shaw (Woodsworth, 2001 et 2003) et sur un traducteur canadien prolifique mais peu connu, Watson Kirkconnell (Woodsworth, 2000b). Parmi ces trois études du début des années 2000, je souhaite revenir ici sur le cas de Valéry, d'une part, car ce poète occupe une place centrale dans ma pensée dès mes études doctorales et, d'autre part, parce que mes recherches actuelles découlent du travail sur le phénomène Poe, entamé dans l'article publié dans le premier numéro de TTR.

6. Voir le texte de Rainier Grutman, "The Self-translator as Author», où sont examinés trois autotraducteurs qui vont plus loin que les «simples» («regular») traducteurs littéraires en adoptant une posture d'auteur («authorial stance») (2018, p. 27). 


\section{Paul Valéry : «Je fus orfèvre de mes chaînes"}

Même si Valéry a traduit relativement peu comparé à ses contemporains, il est néanmoins cité par George Steiner dans son important ouvrage After Babel (1975, p. 269). Lorsque celui-ci dresse la liste de ceux qui ont dit quelque chose de fondamental ou d'original sur la traduction, il mentionne Valéry, au même titre que des traducteurs plus notoires tels que Saint Jérôme, Luther, Pound, Benjamin.

Valéry a signé quelques traductions : des sonnets du poète anglais Gabriel Dante Rossetti et de l'Italien Pétrarque, ainsi qu'un poème de Thomas Hardy, un fragment de Pindare et un article sur la théorie de la gravitation d'Einstein. De façon plus significative, il a publié une traduction de quelques extraits des Marginalia d'Edgar Poe (1980 [1927]) et, vers la fin de sa vie, il a produit une traduction en vers des Bucoliques de Virgile, précédée d'une préface substantielle sur laquelle je reviendrai plus loin.

C'est la traduction de Poe, notamment, qui retient mon attention. Valéry a découvert Edgar Poe en lisant Baudelaire et, de toute évidence, n'a pas lu Poe dans l'original. Il a plutôt pris connaissance des nouvelles de celui-ci grâce au travail de traduction de Baudelaire, dont les recueils de textes traduits sont accompagnés d'introductions élogieuses qui ont contribué à propager une sorte de mythe de Poe en France. L'influence du poète américain, même de seconde main, fut à la fois immédiate et durable. Le jeune Valéry, adoptant le même zèle pour l'Américain que son compatriote Baudelaire, déclare dans une lettre à André Gide du 13 juin 1892 que Poe est «le seul écrivain - sans aucun péché» (1968, vol. 1, p. 1778). À la fin de sa vie, Poe figure encore dans la liste des influences dont Valéry a bénéficié :

En ce qui concerne les influences que j'ai subies, la plus profonde n'est pas celle de Mallarmé : quelques lignes de Poe, la musique de Richard Wagner, l'idée que je me fais de Léonard, et mainte réflexion et lecture scientifiques, ont joué le plus grand rôle dans le développement de ma pensée. (1968, vol. 1, p. 1755)

Quand Valéry décide de traduire Poe, une quarantaine d'années après avoir «subi » ces «influences», il restait peu de choses à traduire (Baudelaire ayant traduit ses nouvelles, et Mallarmé, sa poésie). Valéry choisit donc les Marginalia, réflexions que Poe notait dans les marges des livres qu'il lisait et qu'il fit publier sous forme de livre. Le texte "Quelques fragments des Marginalia, traduits et annotés par Paul Valéry» parut dans la revue Commerce, prestigieuse 
revue dirigée par Valéry en collaboration avec Léon-Paul Fargue et Valery Larbaud. Cette traduction de Poe se présente ainsi : sur les pages de droite, le texte de Poe, traduit en français, et sur celles de gauche les commentaires de Valéry. Curieusement, les manuscrits révèlent que la traduction n'est pas de Valéry: elle est en effet d'une autre main que les quelques corrections de forme et les commentaires où l'on reconnaît bien l'écriture de Valéry. Celui-ci a donc signé une traduction qu'il n'a pas faite, mais qu'il a révisée et commentée. À partir de cette constatation, on peut se demander quelle est la place réelle de la traduction dans les activités intellectuelles de Paul Valéry. J'avancerais que la fonction de la traduction pour Valéry est double : la traduction consiste en un hommage rendu à un écrivain admiré, mais elle est également prétexte. Parmi les Marginalia, qui sont assez considérables, Valéry ne choisit que quelques «fragments». Il choisit de traduire, ou plutôt de faire traduire, les fragments qui sont assez proches de ses propres préoccupations pour servir de point de départ aux commentaires qui paraissent sur la page de gauche. Le premier extrait, par exemple, consiste en une "théorie des notes", ou encore une «théorie des fragments». Valéry étudie deux autres extraits dont l'un porte le sous-titre «De l'Expression» et l'autre «Fatale Supériorité». Dans ses «marginalia» à lui, Valéry formule ses propres idées. La traduction s'insère donc dans un processus de lecture critique et d'écriture, ou plutôt de ré-écriture, à partir du texte lu.

Valéry aborde explicitement la question de la traduction dans la préface à sa traduction des Bucoliques de Virgile. Dans un essai intitulé "Variations sur les Bucoliques», Valéry commente sa propre traduction en y apportant des perspectives intéressantes sur l'art de la traduction. Ce texte, relativement tardif dans la carrière de Valéry ${ }^{7}$, se situe à l'aube de la théorie moderne de la traduction, qui voit le jour vers la fin des années 1940 avec les travaux des linguistes russes et tchèques. Dans ce contexte, les idées de Valéry sur la traduction sont particulièrement originales, pertinentes et même étonnantes par leur avant-gardisme et leur justesse. Dans sa préface, Valéry soulève les difficultés inhérentes à cette traduction, que les commanditaires ont voulue interlinéaire et en vers. En fait, c'est justement la difficulté même qui l'incite à traduire. Valéry aborde ainsi la question de la

7. La traduction des Bucoliques a été faite de 1942 à 1944, à la demande d'un certain $\mathrm{D}^{\mathrm{r}}$ Roudinesco pour une société de bibliophiles; elle parut en 1955, après la mort de Valéry, dans une édition de luxe, accompagnée du texte latin et d'une préface de Valéry (Valéry, 1968, vol. 1, p. 1709). 
motivation du traducteur. Dans le cas d'Edgar Poe, on l'a vu, Valéry traduit - ou fait semblant de traduire - par affinité avec un autre écrivain. Dans le cas de Virgile, par contre, Valéry précise qu'il n'est pas attiré par les thèmes bucoliques de celui-ci : «La vie pastorale m'est étrangère et me semble ennuyeuse», dit-il (1968, vol.1, p. 208-209). Mais Valéry, "orfèvre de [ses] chaînes" tout comme le personnage de Socrate qu'il campe dans Eupalinos (1968, vol. 2, p. 84), accepte de traduire Virgile précisément à cause des problèmes techniques que cela peut lui poser :

Mais enfin, l'espèce de défi que me portaient les difficultés dont j'ai parlé, et les comparaisons mêmes [c'est-à-dire avec tous les autres traducteurs de Virgile] qu'il y avait à craindre, agirent comme des aiguillons et firent que je cédai. (1968, vol. 1, p. 209)

Ce point de vue rejoint un autre thème, celui de la traduction perçue comme pré-texte. En d'autres termes, le travail de traduction sert d'exercice, il permet à l'écrivain de s'entraîner et de perfectionner son art.

L'idée centrale et peut-être la plus originale des "Variations» se fonde sur une comparaison : la traduction est utilisée comme l'un des termes d'une métaphore. La traduction, cependant, n'est plus définie en termes d'autre chose, avec tout ce que cela peut comporter de négatif, comme on l'a vu plus haut; elle est plutôt identifiée à l'écriture.

Écrire quoi que ce soit, aussitôt que l'acte d'écrire exige la réflexion, et n'est pas l'inscription machinale et sans arrêts d'une parole intérieure toute spontanée, est un travail de traduction exactement comparable à celui qui opère la transmutation d'un texte d'une langue dans une autre. (ibid., p. 211; c'est Valéry qui souligne.)

En comparant l'acte d'écrire à la traduction, Valéry inverse la définition métaphorique et, dans ce cas précis, éclaire l'opération traduisante tout en la valorisant.

\section{Le virage fictionnel}

Après un périple de plus de dix ans dans l'administration universitaire, travail non sans intérêt, mais qui m’empêchait d'avancer à fond dans mes travaux traductologiques, je suis revenue à mes premières préoccupations et anciennes amours. À la demande de l'éditeur John Benjamins, je me suis attelée à une tâche assez urgente, celle de mettre à jour Les traducteurs dans l'histoire, ouvrage âgé alors de plus de 
quinze ans ${ }^{8}$. La nouvelle édition sortie, un concours de circonstances m'a mise sur la piste d'un nouveau sujet : ce qu'on appelle depuis une quinzaine d'années le «virage fictionnel» («fictional turn»). Attirée par les recherches sur la "transfiction ${ }^{9}$ et m'intéressant, en même temps, à un certain nombre d'auteurs éminents qui avaient mis la main à la pâte traductive, pour ainsi dire, j'ai repris les sujets qui m'avaient autrefois intéressée tout en m'engageant dans un nouveau domaine de recherche.

Première découverte : l'exposition «Steins Collect», qui eut lieu au Metropolitan Museum de New York en 2012. En regardant les beaux tableaux de Picasso, de Cézanne, de Matisse, entre autres, que la famille Stein avait achetés, conservés ou revendus lorsqu'elle vivait en exil volontaire à Paris dans les premières décennies du vingtième siècle, j’ai appris - non sans étonnement - que Gertrude Stein, cette grande écrivaine avant-gardiste, avait traduit. Affiché au mur, près d'un tableau de Paul Cézanne ${ }^{10}$, se trouvait cet extrait de son Autobiography of Alice B. Toklas:

She had begun not long before as an exercise in literature to translate Flaubert's Trois Contes and then she had this Cézanne and she looked at it and under its stimulus she wrote Three Lives. (Stein, 1961 [1933], p. 34; c'est moi qui souligne)

Apposé sur un autre mur se trouvait un deuxième petit écriteau faisant allusion à une traduction que Stein avait faite de textes du maréchal Pétain, ce qui a piqué ma curiosité tout autant que la référence à Flaubert.

À la même époque, je découvris les textes du New-Yorkais Paul Auster, quasi-polars passionnants peuplés de traducteurs. Il ne faut pas être aussi fin limier que les personnages d'Auster pour découvrir que leur auteur est francophile et ancien traducteur. Et pas

8. En 2012,j'ai fait paraître une nouvelle version de Translators through History. Une version française parallèle des Traducteurs dans l'histoire sort en 2014, traduite sous la direction de Benoit Léger. Une version chinoise, traduite par Guan Xingzhong, paraît en avril 2018 chez China Translation \& Publishing.

9. C'est-à-dire les instances où la traduction figure comme thème, ou les traducteurs comme personnages, dans des œuvres littéraires ou encore au cinéma, et l'étude des implications de ce phénomène pour notre compréhension de l'acte de traduire. Voir Pagano (2000); Arrojo (2002); Delabastita et Grutman (2005).

10. Il s'agit d'un portrait de la femme de Cézanne, intitulé «Madame Cézanne à l'éventail». L'un des quelques portraits de la femme de l'artiste peints entre 1878 et 1888, le tableau appartient maintenant à la Fondation et Collection Emil G. Bührle à Zurich. 
n'importe quel traducteur, car il a traduit des auteurs notables, et non des moindres, comme Jean-Paul Sartre et Paul Éluard.

Gertrude Stein et Paul Auster s'ajoutent donc au répertoire d'écrivains-traducteurs que j'examine. Je poursuis mes recherches, dont les résultats préliminaires sont présentés à un colloque sur la transfiction $^{11}$. Les travaux sur ces deux auteurs aboutissent à une monographie sur les auteurs qui traduisent, où Stein et Auster, en compagnie de Bernard Shaw, sont l'objet d'études de cas qui nous renseignent sur la posture de l'auteur qui traduit (Woodsworth, 2017 et 2018a; sur la notion de "posture littéraire», voir Meizoz, 2007).

\section{Gertrude Stein : la traduction comme « reflet »}

Gertrude Stein a quitté les États-Unis un peu avant la trentaine pour ensuite passer plus de la moitié de sa vie à Paris. Prodigieuse auteure, elle avait toutefois du mal à se faire publier. Son ouvrage The Autobiography of Alice B. Toklas est une mystification litteraire brillante qui consiste en sa propre autobiographie, racontée du point de vue de sa compagne, Alice Toklas. Écrite quand Stein avait presque soixante ans, l'Autobiography fut son premier succès littéraire, dans le monde anglophone aussi bien qu'en France. En essayant de forger sa personnalité d'auteure dans cet ouvrage, Stein se rappelle son premier acte de traduction - celle des Trois Contes survenu quand elle était sur le point d'écrire son premier roman vers 1905 et publié quelques années plus tard à ses propres frais.

En poursuivant mes recherches dans les archives Stein à l'Université Yale, je n'ai trouvé aucune trace de sa prétendue traduction de Flaubert - même pas une ébauche ni un fragment de traduction - alors qu'elle a laissé des quantités de notes, de carnets et de brouillons de toutes sortes de ses autres œuvres. J'en suis venue à la conclusion qu'elle n'a pas traduit Flaubert. Tout au plus, elle a emprunté le format et le titre à Flaubert ${ }^{12}$. La mise en scène de Flaubert, trente ans après la rédaction de Three Lives, peut être

11. «Beyond Transfiction: Translators and (Their) Authors», colloque qui a eu lieu à Tel-Aviv en 2013. La «1st International Conference on Fictional Translators and Interpreters in Literature and Film» avait eu lieu à Vienne en 2011. À la clôture du colloque de Tel-Aviv, j'ai offert de tenir le prochain colloque à l'Université Concordia en 2015, ce qui a donné lieu à un collectif sur la transfiction et d'autres fictions de la traduction, telle que la pseudo-traduction (Woodsworth, 2018b).

12. L'ouvrage de Stein s'appelait d'abord «Three Histories», mais elle a dû changer le titre pour "Three Lives» à la suggestion de l'éditeur, qui publiait aussi des ouvrages historiques proprement dits (Woodsworth, 2017, p. 187 n. 20). 
interprétée dans le contexte de l'objectif de l'Autobiography, soit de renforcer sa crédibilité en tant qu'auteure. Elle se sert de Flaubert pour s'associer à la tradition littéraire française, comme elle évoque Cézanne pour affirmer ses liens avec le mouvement artistique avantgardiste de l'époque. Cette double parenté lui confère son autorité d'écrivaine. La généalogie de Three Lives - qui peut être classée comme une fiction de la traduction ${ }^{13}$ - est très présente dans l'œuvre steinienne, agissant, comme la référence à Poe chez Valéry, en tant que leitmotiv.

Stein a entrepris un autre projet de traduction, plus authentique cette fois, lorsqu'elle a offert au poète surréaliste Georges Hugnet de traduire son cycle de poèmes, Enfances. Comme Hugnet avait traduit ses œuvres à elle, c'était une façon de lui exprimer sa reconnaissance. Pourtant, Stein en tant qu'auteure prend le dessus sur Stein en tant que traductrice : en effet, elle transforme les thèmes sexuels masculins de Hugnet pour créer des poèmes d'amour lesbien, au moyen d'une traduction, ou bien d'une adaptation, très libre. Hugnet appréciait l'approche de Stein; comme il le dit, «[c]e n'est pas une traduction, c'est autre chose, c'est mieux. Je fais plus qu'aimer ce reflet, j'en rêve et je l'admire». Stein, également, concevait la traduction comme un «reflet», mais, en plus, une "expérience riche et intrigante» (correspondance Stein-Hugnet, citée par Woodsworth, 2017, p. 189 n. 36). Finalement, c'est un problème de mise en page qui a mis fin à l'amitié entre le poète et sa traductrice : le nom de Hugnet figurait en haut de la page, avec le nom de Stein, en tant que traductrice, en caractères plus petits sous le nom de l'auteur. Elle s'offusqua, car elle pensait avoir été «collaboratrice» au lieu de simple traductrice.

Pendant la Deuxième Guerre mondiale, Stein a aussi traduit les discours de Pétain, à la demande de son ami, acolyte et traducteur Bernard Faÿ, intellectuel estimé qui fut condamné après la Guerre pour «indignité nationale». La participation de cette Américaine juive dans une telle entreprise propagandiste en faveur du régime de Vichy a provoqué des réactions négatives, mais la situation est loin d'être claire. Stein, assistée par Alice, a rempli trois cahiers manuscrits

13. Cette traduction fictive, comme celle de Valéry, peut faire penser au phénomène de "pseudo-traduction", sauf que ce dernier terme est généralement réservé aux traductions présumées pour lesquelles il n'y a pas d'original (Toury, 1995, p. 40). Dans ces deux cas, il y a bel et bien un original, que l'auteur prétend avoir traduit. La désignation «fiction de la traduction", par conséquent, s'applique mieux à ce genre de mystification littéraire. 
de ses versions anglaises des discours de Pétain, mais la traduction ne vit jamais le jour. L'introduction de Stein est significative. En effet, Stein ne se comporte par comme d'autres traducteurs le font souvent : au lieu de se servir de l'espace paratextuel pour présenter l'œuvre ou s'exprimer sur l'acte de traduction lui-même, elle y fait l'éloge de Pétain, qu'elle compare à George Washington, vraisemblablement afin de rehausser l'image du chef de l'État français auprès du public américain visé (Stein, 1996). Stein n’a jamais parlé explicitement de cette traduction, qui l'a occupée pendant près de deux ans dans les années 1940, bien qu'elle en dise long sur ces années d'exil dans sa maison de campagne à la même période. On ne connaît pas, par conséquent, sa véritable motivation ou "pulsion de traduire». Cette traduction, on peut spéculer, était un geste d'amitié, une forme de reconnaissance envers Faÿ, qui aurait permis à Gertrude et Alice de survivre aux événements catastrophiques de l'époque. ${ }^{14}$

Elle s'est toutefois prononcée sur son expérience comme traductrice des poèmes de Hugnet, qu'elle décrit dans une conférence qu'elle prononça pendant sa célèbre tournée des États-Unis en 1934-1935: "a very queer thing was happening [...] I finished the whole thing not translating but carrying out an idea which was already existing» (cité par Woodsworth, 2017, p.95; je souligne). En traduisant, Stein découvrait une nouvelle manière d'écrire, la traduction lui servant de préparation à son futur travail de poète.

\section{Paul Auster : la traduction comme exercice et découverte}

Paul Auster s'était intéressé à la langue, à la littérature et à la culture françaises dès son plus jeune âge. Comme d'autres écrivains américains avant lui, il est allé passer quelques années en France, où il apprit le métier d'écrivain tout en vivant de ses traductions. Sa passion pour les poètes français remontait à ses années universitaires, pendant lesquelles il avait fait paraitre une "Little Anthology of Surrealist Poems». À Paris, il rencontra des poètes et se lia d'amitié avec eux. Le résultat fut une autre anthologie, massive cette fois, de la poésie française du vingtième siècle (Auster, 1982), travail «d'amour» qui rassemblait les poètes français traduits par d'éminents écrivains de

14. Ces traductions, inachevées et jamais publiées, sont examinées en détail dans mon ouvrage (Woodsworth, 2017). Seule l'introduction est parue, aux États-Unis (Stein, 1996), lorsqu'une doctorante américaine l'a découverte dans les archives de la maison d'édition Random House et l'a publiée avec un commentaire (Van Dusen, 1996). 
langue anglaise tels que Samuel Beckett et T.S. Eliot, et d'illustres traducteurs. Traducteur de plusieurs poèmes lui-même, Auster est à l'origine des choix difficiles inhérents à toute anthologie ${ }^{15}$. En tant que directeur du recueil, comme il le signale dans son introduction assez savante d'ailleurs - il agit en tant que « marieur» (" matchmaker») entre poètes et traducteurs (ibid., p. xlviii).

À une certaine époque, Auster a les moyens financiers de laisser tomber ses projets de traduction, parfois ennuyeux, et de se consacrer à la création littéraire. Dans son premier roman, City of Glass, le personnage principal, Daniel Quinn, est un ancien traducteur. Quand Quinn rencontre le personnage fictif qui, comme l'auteur, s'appelle Paul Auster, de quoi discutent-ils? De la traduction et du célèbre roman de Cervantes, Don Quichotte, considéré comme un exemple notoire de pseudo-traduction. Les traducteurs apparaissent dans plusieurs de ses romans. Dans The Book of Illusions (Le Livre des illusions; 2002), par exemple, le protagoniste Zimmer traduit les Mémoires d'outre-tombe de Chateaubriand ${ }^{16}$. Le monde fictif d'Auster constitue une «salle des miroirs» de la traduction.

Celui qui est salué comme «décathlonien littéraire» par un journaliste rend hommage aux traducteurs, tout en soulignant les misères de la traduction d'un côté et ses splendeurs de l'autre (voir Ortega y Gasset, 1992). Comme il l'affirme : «[t]ranslators are the shadow heroes of literature, the often forgotten instruments that make it possible for different cultures to talk to one another» (cité par Woodsworth, 2017, p. 121). Il a aussi signalé l'importance de la traduction dans sa propre évolution comme écrivain :

It was part of my development as a writer to translate and I found it thrilling and helpful ... a way of feeling more comfortable with a pen in my hand looking at a blank page. (entretien personnel avec Paul Auster, juin 2013)

15. Lanthologie débute avec "Zone» d'Apollinaire même si elle contient des œuvres de poètes français plus anciens, sans doute à cause de sa signification comme chant de la modernité. Le poème s'ouvre par «À la fin tu es las de ce monde ancien », vers qu'Auster reprend, dans son introduction, tel que traduit par Beckett : "In the end you are weary of the ancient world» (1982, p. xxxiv).

16. Chateaubriand est connu pour sa traduction de Paradise Lost de Milton et pour celle des "poèmes d'Ossian», exemple illustre de pseudo-traduction. Auster reproduit un extrait de la traduction sur laquelle travaille Zimmer, passage où Napoléon est justement en train de lire les traductions «ossianiques». 
Comme chez Valéry et Stein, la traduction sert chez Paul Auster non seulement d'exercice, mais aussi de thème. Elle est perçue comme un acte de découverte et d'amour : "not simply as a literary exercise, but as an act of discovery and passion» (Auster, 1982, p. xxx).

\section{Idra Novey : traductrice aérienne}

Dans son roman Ways to Disappear (2016b), l'écrivaine et traductrice new-yorkaise Idra Novey met une traductrice et la traduction en scène. Traductrice - de la Brésilienne Clarice Lispector notamment - avant de devenir romancière, Novey évoque dans son roman la fameuse expression traduttore, traditore, qu'elle appelle un "tired, tortured Italian cliché»(Novey, 2016b, p. 97). Son personnage de traductrice n'en est pas moins hanté par l'impossibilité de traduire. Le roman tourne autour de l'infidélité conjugale, qui sert de toile de fond à la traduction conçue comme trahison et transgression. En même temps, l'acte de traduire est vu comme point de départ à des tentatives de création, des formes embryonnaires d'écriture qui pavent la voie à l'écriture originale. Comme le fait remarquer Novey :

Translation begins with the prefix "trans" for a reason. Like transcendence and transformation, it requires an acceptance of progressing with uncertainty, which is essential for authors [...] To begin writing after translating is to begin airborne - suspended between languages - a reckless place I'd like to believe leaves a writer's mind particularly open to innovation. (2016a, s.p.)

Cet exemple récent, qui s'ajoute aux études de cas que j'ai évoqués rapidement, montre encore une fois que la traduction sert de préparation à l'écriture, et que la transfiction sert à rendre encore plus floues les frontières traditionnelles entre l'écriture et la traduction, tout en nous permettant d'étendre notre compréhension des multiples enjeux de la traduction littéraire dans le monde actuel.

Les écrivains qui traduisent ont tendance à se servir de la traduction comme d'un tremplin, qui permet de concevoir une nouvelle façon d'écrire. À cause d'une longue tradition de servilité, les traducteurs hésitent souvent à adopter une posture d'auteur et agissent plutôt avec humilité - qu'elle soit réelle ou feinte. Les écrivains, par contre, même en acceptant de traduire les textes des autres, restent auteurs, affirmant leur autorité. Qu'ils commentent leur traduction, comme le fait Valéry dans ses «Variations» ou Stein dans son «Autobiographie» et d'autres écrits, ils prennent la parole 
en tant qu'auteurs. En d'autres mots, ils profitent de l'espace de l'écrivain, où ils se permettent de construire leur personne en tant qu'écrivain, et de raconter l'histoire de leurs traductions d'une façon qui est différente de celle des traducteurs «ordinaires».

Dans mes études récentes ${ }^{17}$, j'ai essayé de démontrer les diverses motivations et approches de certains traducteurs et traductrices qui ont usé et abusé de la notion de «traduction» de façons variées, et parfois contradictoires. En adoptant des stratégies de traduction, ils peuvent se permettre de traduire de façon plus libre. Il leur arrive de profiter de leur travail de traducteurs et de traductrices pour exprimer leurs propres idées, leurs prises de position esthétiques et leurs préoccupations personnelles. Ils peuvent ainsi transgresser les frontières de la traduction proprement dite, comme on l'a vu en évoquant les cas de traduction fictive. Mes derniers travaux ont comme but de brosser un tableau plus nuancé de ces questions. On peut émettre l'hypothèse suivante, pourtant : qu'ils soient saboteurs du processus de traduction ou non, les écrivains qui traduisent contribuent à redorer l'art de la traduction, trop longtemps considéré comme un art secondaire, inférieur, du fait même qu'ils ont pris le temps de se consacrer à la traduction en tant qu'acte littéraire de plein droit.

\section{References}

Arrojo, Rosemary (2002). «Writing, Interpreting, and the Power Struggle for the Control of Meaning: Scenes from Kafka, Borges, and Kosztolányi». In M. Tymoczko et E. Gentzler, dir. Translation and Power. Amherst, University of Massachusetts Press, p. 63-79.

Auster, Paul, dir. (1982). The Random House Book of Twentieth-Century French Poetry. New York, Random House.

Auster, Paul (1987). City of Glass. In The New York Trilogy. New York, Faber $\&$ Faber.

Auster, Paul (2002). The Book of Illusions. New York, Henry Holt.

Bassnett, Susan et André Lefevere (1990). "Introduction: Proust's Grandmother and the Thousand and One Nights. The "Cultural Turn" in Translation Studies». In S. Bassnett and A. Lefevere, dir. Translation, History, and Culture. London, Cassell, p. 1-13.

Baudelaire, Charles (1932). «Histoire des Histoires extraordinaires». In Histoires extraordinaires. Paris, Conard.

Berman, Antoine (1984). L'Épreuve de l'étranger. Culture et traduction dans l'Allemagne romantique. Paris, Gallimard.

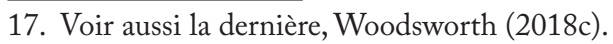


Berman, Antoine (1985). Les tours de Babel : essais sur la traduction. Mauvezin, Trans-Europ-Repress.

Chesterman, Andrew (2009). «The Name and Nature of Translator Studies». Hermes: Journal of Language and Communication Studies, 42, p. 13-22.

Delabastita, Dirk et Rainier Grutman (2005). «Introduction. Fictional Representations of Multilingualism and Translation». In D. Delabastita et R. Grutman, dir. Fictionalising Translation and Multilingualism, Linguistica Antverpiensia, 4, p. 11-34.

Delisle, Jean (s.d.). «Historique de l'Association canadienne de traductologie». Disponible à <http://act-cats.ca/fr/propos/historiqueet-buts/> [consulté le 30 juillet 2018].

Delisle, Jean (2017). La traduction en citations. Florilège. $2^{\mathrm{e}}$ éd. Ottawa, Les Presses de l'Université d'Ottawa.

Delisle, Jean et Judith Woodsworth (2012). Translators through History. Amsterdam/Philadelphie, John Benjamins.

Delisle, Jean et Judith Woodsworth (2014). Les traducteurs dans l'bistoire. Trad. Benoit Léger. Québec, Presses de l'Université Laval.

Galli, Pauline (2012). «De Poe à Mallarmé, de Mallarmé à Poe : traduction, édition, création ». TTR, 25, 2, p. 143-165.

Gouanvic, Jean-Marc, dir. (1988). «Traduction et culture(s)». TTR, 1, 1.

Grutman, Rainier (2018). «The Self-translator as Author. Modern Selffashioning and Ancient Rhetoric in Federman, Lakhous and De Kuyper». In J. Woodsworth, dir. The Fictions of Translation. Amsterdam/ Philadelphie, John Benjamins, p. 15-30.

Guillerm, Luce (1980). «L'auteur, les modèles, et le pouvoir ou la topique de la traduction au XVIe siècle en France». Revue des sciences humaines, t. LII, 180, octobre-décembre, p. 15.

Hermans, Theo (1985). «Metaphor and Imagery in the Renaissance Discourse on Translation». In T. Hermans, dir. The Manipulation of Literature. New York, St. Martin's Press, p. 107-108.

Holmes, James S. (2004 [1972]). «The Name and Nature of Translation Studies». In L. Venuti, dir. The Translation Studies Reader. $2^{\mathrm{e}}$ éd. Londres et New York, Routledge, p. 180-192.

Meizoz, Jérôme (2007). Postures littéraires. Mises en scène modernes de l'auteur. Genève, Slatkine Érudition.

Novey, Idra (2016a). «Writing while Translating». Literary Hub, 25 February. Disponible à <https://lithub.com/writing-while-translating/> [consulté le 15 février 2018].

Novey, Idra (2016b). Ways to Disappear. New York, Little, Brown and Company.

Ortega y Gasset,José (1992). «The Misery and the Splendor of Translation ». Trad. Elizabeth Gamble Miller. In R. Schulte et J. Biguenet, dir. Theories of Translation. An Anthology of Essays from Dryden to Derrida. Chicago, University of Chicago Press, p. 93-112. 
Pagano, Adriana S. (2000). "Sources for Translation Theory: Fiction in Latin America». ATA Chronicle, 29, 4, p. 38-44.

Poe, Edgar et Paul Valéry (1980). Fragments des Marginalia traduits et commentés par Paul Valéry. Montpellier, Fata Morgana.

Stein, Gertrude (1961 [1933]). The Autobiography of Alice B. Toklas. New York, Vintage Books.

Stein, Gertrude (1996). «Introduction to the Speeches of Maréchal Pétain». W. Van Dusen, dir. Modernism/modernity, 3, 3, p. 93-96.

Steiner, George (1975). After Babel: Aspects of Language and Translation. Oxford, Oxford University Press.

Toury, Gideon (1995). Descriptive Translation Studies and Beyond. Amsterdam/Philadelphie, John Benjamins.

Valéry, Paul (1927). «Quelques fragments des Marginalia, traduits et annotés par Paul Valéry». Commerce, 14, hiver, p. 11-41. [Réédité comme Poe et Valéry, 1980.]

Valéry, Paul (1968). Euvres. J. Hytier, dir. Paris, Gallimard, 2 vols.

Van Dusen, Wanda (1996). «Portrait of a National Fetish: Gertrude Stein's "Introduction to the Speeches of Maréchal Pétain" (1942)».Modernism/ Modernity, 3, 3, p. 69-92.

Woodsworth, Judith (1977). Valéry et Poe: le délire de la lucidité. Thèse de doctorat, Département de langue et littérature françaises, Université McGill. Inédit.

Woodsworth, Judith (1988). «Traducteurs et écrivains : vers une redéfinition de la traduction littéraire». TTR, 1, 1, p. 115-125.

Woodsworth, Judith (1990). "Metaphor and Theory: Describing the Translation Process». In P. Chaffey et al., dir. Translation Theory in Scandinavia. Proceedings of SSOTT III (Scandinavian Symposium on Translation Theory, 1988). Oslo, University of Oslo, p. 281-299.

Woodsworth, Judith (2000a), «Fragments d'une théorie de la traduction : Paul Valéry traducteur ». In M. Bouchard et al., dir. Mélanges à la mémoire de Jean-Claude Morisot. Littératures, 21-22. Montréal, Université McGill, p. 245-263.

Woodsworth, Judith (2000b). «Watson Kirkconnell and the "Undoing of Babel": A Little-Known Case in Canadian Translation History». Meta, 45, 1, p. 13-28.

Woodsworth, Judith (2001). «In the Looking Glass: Bernard Shaw on and in Translation». In S. Petrilli, dir. Athanor, «Lo stesso altro», 4, p. 128145.

Woodsworth, Judith (2003). «In the Looking Glass: Bernard Shaw on and in Translation». In S. Petrilli et A. Ponzio, dir. Translation Translation. Amsterdam/New York, Rodopi, p. 531-551.

Woodsworth, Judith (2017). Telling the Story of Translation: Writers Who Translate. London, Bloomsbury. 
Woodsworth, Judith (2018a). "Gertrude Stein and the Paradox of Translation». In J. Woodsworth, dir. The Fictions of Translation. Amsterdam/Philadelphie, John Benjamins, p. 31-48.

Woodsworth, Judith, dir. (2018b). The Fictions of Translation. Amsterdam/ Philadelphie, John Benjamins.

Woodsworth, Judith (2018c). «Writers as Translators». In K. Washbourne et B. Van Wyke, dir. The Routledge Handbook of Literary Translation. Londres et New York, Routledge, p. 369-381.

\author{
Judith Woodsworth \\ Département d'études françaises \\ Université Concordia \\ Montréal (Québec), Canada \\ judith.woodsworth@concordia.ca
}

